\title{
Too old to have a tumour of the young?
}

\section{Case report}

A 38-year-old Caucasian male presented to hospital with a 2-week history of progressive exertional dyspnoea and intermittent left pleuritic chest pain, which was not relieved by analgesia.

The patient denied cough, sputum production, haemoptysis, wheeze, weight loss, vomiting, altered bowel habits, urinary symptoms, recent trauma, recent travel and immobility. He had a history of hypertension, allergic rhinitis, obesity and chronic bilateral leg oedema.

He had a 25 pack-year smoking history, drank minimal alcohol and was taking atenolol and aspirin. There was no family history.

\section{Examination and investigations}

Examination revealed that the patient was obese, febrile $\left(37.7^{\circ} \mathrm{C}\right)$ and hypoxaemic (pulse oximetry of $89 \%$ on room air), with a respiratory rate of 25 breaths per min, blood pressure of $95 / 65 \mathrm{mmHg}$ and a pulse of 120 beats per min (sinus tachycardia).

The patient had clinical signs consistent with a left pleural effusion, which was confirmed by chest radiography. There was no wheeze or crepitations, and heart sounds were normal. His jugular venous pressure was not raised and there was no palpable lymphadenopathy. Abdominal examination was unremarkable and the limbs did not demonstrate signs of deep vein thrombosis.

Pleural aspiration revealed bloodstained fluid $(\mathrm{pH} 7.19)$ and negative cytology and culture. Bronchoscopy was not undertaken owing to the hypoxaemia and radiological findings. As the patient had swinging fevers, an infective cause was initially suspected and he was there fore commenced on broad spectrum intravenous antibiotics.

\section{Task 1 \\ What can be seen on the representative CT slice?}

\section{Task 2}

Can you explain what can be seen from the findings of the CT-guided biopsy?
A contrastenhanced computed tomography (CT) scan of the chest revealed a large left pleural effusion with no evidence of pulmonary embolus, broncho-stenosing lesion or empyema.

Owing to persistent fever and pain, a second CT scan was performed after partial pleural drainage. A representative slice is shown in figure 1. A CT-guided biopsy was also performed (figure 2).

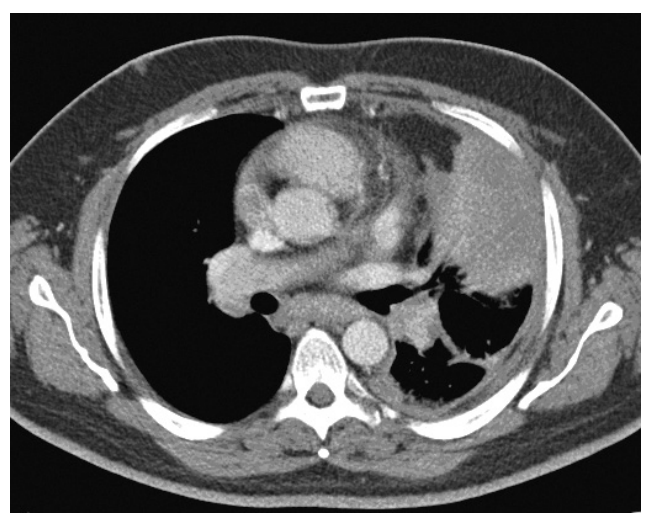

Figure 1

A representative slice from a second CT scan.

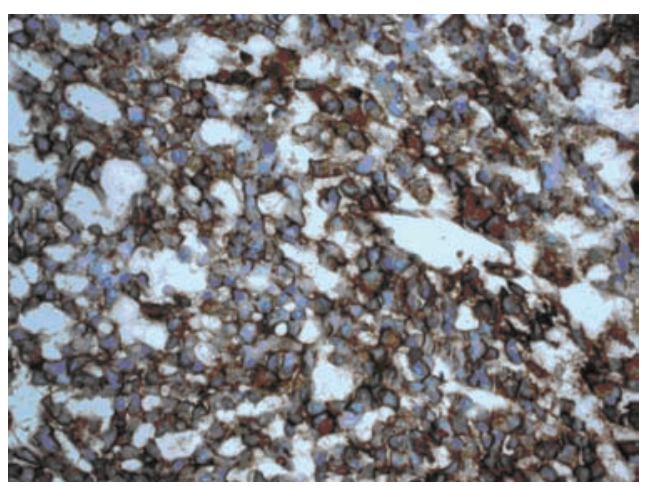

Figure 2

Finding from CT-guided biopsy.

\section{Task 3}

Please suggest a diagnosis for this patient.

\section{Task 4}

How would you treat this patient?
D. Craig 1

C.M.Ohri ${ }^{2}$

R. Benson ${ }^{3}$

C.J. Hunter ${ }^{1}$

1Dept of Respiratory Medicine, Peterborough District Hospital, Peterborough, 2Dept of Respiratory Medicine, Glenfield Hospital, Leicester, and '3ept of Oncology, Addenbrooke's Hospital, Cambridge, UK.

\section{Correspondence:}

C. Hunter

Peterborough District Hospital Thorpe Road Peterborough PE3 6DA

UK

Fax: 441733875287

E-mail:cameron.hunter@ pbh-tr.nhs.uk 


\section{Answer 1}

The CT scan was reviewed and demonstrated a large thoracic mass surrounded by a small amount of fluid.

\section{Answer 2}

CT-guided biopsy revealed cellular necrosis, with negative staining for CD99 antigen.

\section{Answer 3}

The results are suggestive of an Askin tumour, a rare primitive neuroectodermal tumour (PNET) that usually affects children or young adults.

\section{Answer 4}

The patient was treated with six cycles of chemotherapy with vincristine, ifosfamide, doxorubicin and etoposide. Unfortunately, imaging at the end of the chemotherapy showed little response and, despite an attempted resection, the patient died of his disease 7 months after diagnosis.

\section{Discussion}

In 1979, Askin et al. [1] described a series of malignant small round-cell tumours of the chest wall occurring in children and young adults. Askin's tumour derives from the Ewing's family of tumours and is a PNET of the chest wall. A characteristic chromosomal translocation, $(11 ; 22)(q 24 ; q 12)$ can be detected in $90 \%$ of PNET and Ewing's sarcomas [2].

The most common mode of presentation is with a thoracic mass accompanied by chest wall pain $[3,4]$ or with fever, weight loss and dyspnoea. Initial radiographic appearances typically show large soft tissue masses in the chest, frequently accompanied by an ipsilateral pleural effusion. Askin tumours are highly malignant and most are advanced at the time of presentation. Imaging often shows rib destruction and a large soft tissue mass, which may occasionally contain calcification [5]. The affected ribs show increased uptake on bone scintigrapy scanning and the diagnosis is made by biopsy. Optimal treatment is with multi-drug chemotherapy and subsequent resection of the rib and any underlying residual pleural thickening or tumour and/or radiotherapy.

Prognosis for the Ewing's family of tumours is related to tumour volume, presence of metastases, tumour resectability, presence of fever and anaemia. A significant response to preoperative therapy is also a favourable sign. Younger patients also seem to have a better prognosis [6].

Traditionally, these tumours have been described in children and adolescents [1], but the present case reinforces the notion that Askin tumours, although rare, can occur in adults. It is also possible that, in the past, lung cancers have been incorrectly diagnosed in adults with a PNET [7].

\footnotetext{
References

1. Askin FB, Rosai J, Sibley RK, Dehner LP, McAlister WH. Malignant small cell tumor of the thoracopulmonary region in childhood: a distinctive clinicopathologic entity of uncertain histogenesis. Cancer 1979; 43: 2438-2451.

2. Amatruda JF, Tran K, Mantel M, Singer S, Demetri G. Askin tumor. J Clin Oncol 1998; 16: 1997-1998.

3. Takanami I, Imamura T. The treatment of Askin tumor: Results of two cases. J Thorac Cardiovasc Surg 2002; 123: 391-392.

4. Miller JA, Tomkovich K, Romberger C. Primitive neuroectodermal tumour of the chest wall (Askin tumour): (T and roentgenographic findings in a 51-year-old male. Respir Med 1995; 89: 705-708.

5. Dick EA, McHugh K, Kimber C, Michalski A. Imaging of non-central nervous system primitive neuroectodermal tumours: diagnostic features and correlation with outcome. Clin Radiol 2001; 56: 206-215.

6. Cotterill SJ, Ahrens S, Paulussen M, et al. Prognostic factors in Ewing's tumor of bone: analysis of 975 patients from the European Intergroup Cooperative Ewing's Sarcoma Study Group. J Clin Oncol 2000; 18: 3108-3114.

7. Takagi-Takahashi Y, Shijubo N, Yamada G, et al. Peripheral primitive neuroectodermal tumor of the chest wall of a 69-year-old man. Intern Med 2004; 43: 578-581.
} 